\title{
MIMO-OFDM Channel Estimation using Pilot Carries
}

\author{
Kala Praveen Bagadi \\ Ph.D Student \\ Dept. of Electrical Engineering \\ NIT Rourkela, India
}

\author{
Prof. Susmita Das \\ Associate Professor \\ Dept. of Electrical Engineering \\ NIT Rourkela, India
}

\begin{abstract}
A multiple-input multiple-output (MIMO) communication system combined with the orthogonal frequency division multiplexing (OFDM) modulation technique can achieve reliable high data rate transmission over broadband wireless channels. Channel state information for both single-input single-output (SISO) and MIMO systems based on pilot aided arrangement is investigated in this paper. The estimation of channel at pilot frequencies with conventional Least Square (LS) and Minimum Mean Square (MMSE) estimation algorithms is carried out through Matlab simulation. The performance of MIMO OFDM and SISO OFDM are evaluated on the basis of Bit Error Rate (BER) and Mean Square Error (MSE) level. Further enhancement of performance can be achieved through maximum diversity Space Time Block Coding (STBC) and Maximum Likelihood Detection at transmission and reception ends respectively. MMSE estimation has been shown to perform much better than LS but is more complex than LS for the MIMO system using pilot carriers.
\end{abstract}

\section{Keywords}

Channel Estimation, MIMO-OFDM, Pilot carriers, Diversity, Spatial Multiplexing, Space time coding, Rayleigh fading.

\section{INTRODUCTION}

OFDM (Orthogonal Frequency Division Multiplexing) is becoming a very popular multi-carrier modulation technique for transmission of signals over wireless channels. OFDM divides the high-rate stream into parallel lower rate data and hence prolongs the symbol duration, thus helping to eliminate Inter Symbol Interference (ISI). It also allows the bandwidth of subcarriers to overlap without Inter Carrier Interference (ICI) as long as the modulated carriers are orthogonal. OFDM therefore is considered as an efficient modulation technique for broadband access in a very dispersive environment.

In this new information age, high data rate and strong reliability in wire-less communication systems are becoming the dominant factors for a successful exploitation of commercial networks. MIMO-OFDM (multiple input multiple outputorthogonal frequency division multiplexing), a new wireless broadband technology, has gained great popularity for its capability of high rate transmission and its robustness against multi-path fading and other channel impairments. The arrangement of multiple antennas at the transition end and reception end results increase in the diversity gain refers the quality of signal and multiplexing gain refers the transmission capacity. Space time block coding used in this paper to transmit multiple copies of a data stream across a number of antennas and to exploit the various received versions of data to improve reliability of data transfer.

The major challenge faced in MIMO-OFDM systems is how to obtain the channel state information accurately and promptly for coherent detection of information symbols. The channel state information can be obtained through training based, blind and semi blind channel Estimation. The blind channel estimation is carried out by evaluating the statistical information of the channel and certain properties of the transmitted signals [3]. Blind Channel Estimation has its advantage in that it has no overhead loss; it is only applicable to slowly time-varying channels due to its need for a long data record. In training based channel estimation algorithms, training symbols or pilot tones that are known a priori to the receiver, are multiplexed along with the data stream for channel estimation [4]. Semi-blind channel technique is hybrid of blind and training technique, utilizing pilots and other natural constraints to perform channel estimation.

The training-based method channel estimation can be performed by either block type pilots where pilot tones are inserted into all frequency bins within periodic intervals of OFDM blocks or by comb pilots where pilot tones are inserted into each OFDM symbol symbols with a specific period of frequency bins. The block type pilot channel estimation, has been developed under the assumption of slow fading channel; this assumes that the channel transfer function is not changing very rapidly it can be constant over transmission of few OFDM symbols. The comb-type pilot channel estimation has been introduced in case where the channel changes even in one OFDM block. The comb-type pilot channel estimation consists of algorithms to estimate the channel at pilot frequencies and interpolation is used to find the channel at signal frequencies [5]. The interpolation of the channel for comb-type based channel estimation can be depend on linear interpolation, low-pass interpolation and spline cubic interpolation. Further, this signal model is transformed into a linear form suitable for the LS (leastsquare) and MMSE (minimum mean square error) estimation algorithm. MMSE has been shown to perform much better than LS but more complex than LS. We can use optimal low rank MMSE estimator to reduce complexity. And finally we can conclude that MMSE is an optimal channel estimator in the sense of achieving the minimum MSE (mean squared error). The organization of the paper is as follows. Section II and III describe the system models of SISO \& MIMO OFDM systems respectively. Section IV provided the performances of LS and LMMSE algorithms in both the systems and simulation results are presented in Section V Conclusion is given in Section VI. 


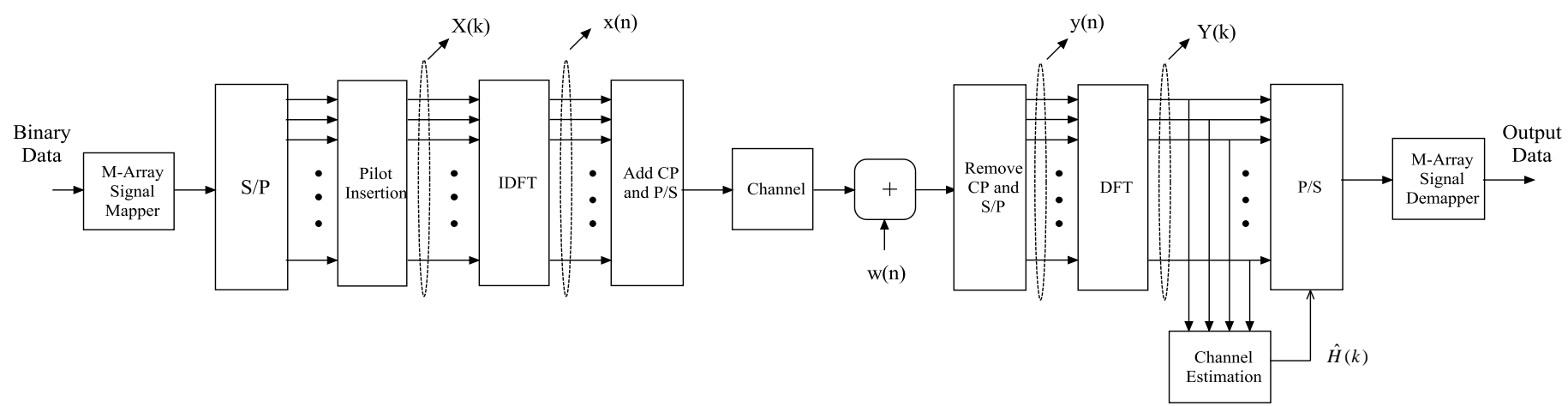

Figure 1. SISO OFDM System Block Diagram

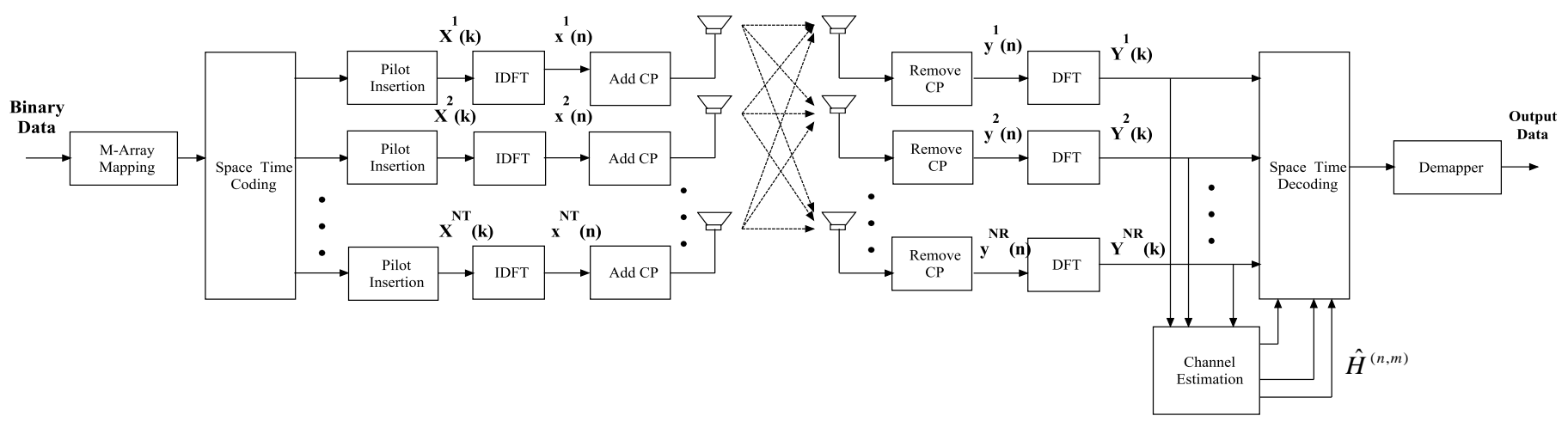

Figure 1. SISO OFDM System Block Diagram 


\section{SISO OFDM SYSTEM MODEL}

The SISO OFDM system block based on Training sequence is given in Fig. 1. The binary data is first grouped and mapped according to the modulation in "signal mapper". Dependent on the channel condition like fading, different base modulation modes such as BPSK (binary phase shift keying), QPSK (quadrature phase shift keying) and QAM are used to boost the data rate. After modulation the symbol rate reduced to $R=(R /$ $\log _{2}{ }^{\mathrm{M}}$ ), where $\mathrm{M}$ is constellation size. Then this serial data is fed to serial to parallel convertor. This reduces data rate by $\mathrm{N}$ times, where $\mathrm{N}$ is number of parallel streams. Each of parallel stream constitutes tiny bandwidth in the spectrum. So these streams almost undergo flat fading in the channel. This is the greatest advantage of OFDM. After inserting pilots either to all subcarriers with a specific period of blocks or within a uniform period of frequency bins in all blocks, IDFT block is used to transform the data sequence of length into time domain signal with the following equation:

$$
\begin{aligned}
& x(n)=\operatorname{IDFT}\{X(k)\} \quad n=0,1,2 \ldots . N-1 \\
= & \sum_{k=0}^{N-1} X(k) e^{j(2 \pi k n / N)}
\end{aligned}
$$

where $w(n)$ is Additive White Gaussian Noise (AWGN) and $m$ is the total number of propagation paths and $\tau_{m}$ is delay spread. At the receiver's end, after passing to discrete domain through A/D and low pass filter, guard time is removed the signal $y^{g}\left(n+n_{g}\right)$ for $-N_{g} \leq n \leq N-1$ will become as $y(\mathrm{n})$ for $n=0,1,2 \ldots . . N-1$

Then $\mathrm{y}(\mathrm{n})$ is sent to DFT block for the following operation:

$$
Y(k)=\operatorname{DFT}\{y(n)\} \quad k=0,1,2 \ldots . . N-1
$$

As a matter of convenience we can write the entire operation as:

$$
Y(k)=X(k) H(k)+W(k)
$$

Where $X(k)=\operatorname{DFT}\{x(n)\}$ and $W(k)=\operatorname{DFT}\{w(n)\}$.

Then the binary information data is obtained back in "signal demapper" block. where $N$ is DFT length. Following IDFT block, guard time, which is chosen to be larger than the expected delay spread, is inserted to prevent inter-symbol interference. This guard time is a copy of the last part which is prepended to OFDM symbol. This makes the transmitted symbol periodic, which plays a decisive role in identifying frames correctly, so as to avoid ISI and intercarrier interference (ICI). The resultant OFDM symbol is given as follows:

$$
\begin{gathered}
\left.x^{g}(n)=x\left(n_{g}+n\right)\right) \quad n_{g}=-N_{g}, N_{g}+1 \ldots . .1 \\
n=0,1,2 \ldots . . N-1
\end{gathered}
$$

where $N_{g}$ is the length of the guard interval. The transmitted signal will pass through the frequency selective time varying fading channel with additive noise. Then the received signal is

$$
y^{g}(n)=\left\{x^{g}(n) \otimes h(n)\right\}+w(n)
$$

The channel response $h$ can be represented by

$$
h(n)=\frac{1}{\sqrt{N}} \sum_{m} \alpha_{m} e^{-j \frac{\pi}{N}\left(k+(N-1) \tau_{m}\right)} \frac{\sin \left(\pi \tau_{m}\right)}{\sin \left(\frac{\pi}{N}\left(\tau_{m}-k\right)\right)}
$$

\section{MIMO OFDM SYSTEM}

Fig. 2 depicts a high level block diagram of the MIMO OFDM system. We consider MIMO-OFDM systems with two transmit antennas and two receive antennas. The total number of subcarriers is N. Basically, the MIMO-OFDM transmitter has $N_{t}$ parallel transmission paths which are very similar to the single antenna OFDM system, each branch performing serial-to-parallel conversion, pilot insertion, $\mathrm{N}$-point IFFT and cyclic extension before the final TX signals are up-converted to RF and transmitted. It is worth noting that the channel encoder and the digital modulation, in some spatial multiplexing systems, can also be done per branch, where the modulated signals are then space-time coded using the Alamouti algorithm [6] before transmitting from multiple antennas [7] not necessarily implemented jointly over all the $N_{t}$ branches. Subsequently at the receiver, the $\mathrm{CP}$ is removed and $\mathrm{N}$-point FFT is performed per receiver branch. Next, the transmitted symbol per TX antenna is combined and outputted for the subsequent operations like digital demodulation and decoding. Finally all the input binary data are recovered with certain BER. 
As a MIMO signalling technique, $N_{t}$ different signals are transmitted simultaneously over $N_{t} \mathrm{X} N_{r}$ transmission paths and each of those $N_{r}$ received signals is a combination of all the $N_{t}$ transmitted signals and the distorting noise. It brings in the diversity gain for enhanced system capacity as we desire. Meanwhile compared to the SISO system, it complicates the system design regarding to channel estimation and symbol detection due to the hugely increased number of channel coefficients. The data stream from each antenna undergoes OFDM Modulation. The Alamouti Space Time Block Coding (STBC) scheme has full transmit diversity gain and low complexity decoder, with the encoding matrix represented as referred in [8] as

$$
\begin{aligned}
& X=\left[\begin{array}{cc}
X_{1} & -X_{2}^{*} \\
X_{2} & X_{1}^{*}
\end{array}\right] \\
& X_{1}=\left(X[0]-X^{*}[1] X[2]-X^{*}[3] \ldots \ldots-X^{*}[N-1]\right) \\
& X_{2}=\left(X[1] \quad X^{*}[0] X[3] \quad X^{*}[2] \ldots \ldots \ldots X^{*}[N-2]\right)
\end{aligned}
$$

The vectors $X_{1}$ and $X_{2}$ are modulated using the inverse fast Fourier transform (IFFT) and after adding a cyclic prefix as a guard time interval, two modulated blocks $X^{g}{ }_{1}$ and $X^{g}{ }_{2}$ are generated and are then transmitted by the first and second transmit antennas respectively. Assuming that the guard time interval is more than the expected largest delay spread of a multipath channel. The received signal will be the convolution of the channel and the transmitted signal. Assuming that the channel is static during an OFDM block, at the receiver side after removing the cyclic prefix, the FFT output as the demodulated received signal can be expressed as

$$
\left[\begin{array}{c}
Y_{1} \\
Y_{2} \\
\vdots \\
Y_{N_{R}}
\end{array}\right]=\left[\begin{array}{cccc}
H_{1,1} & H_{1,2} & \cdots & H_{1, N_{T}} \\
H_{2,1} & H_{2,2} & \cdots & H_{2, N_{T}} \\
\vdots & \vdots & \ddots & \vdots \\
H_{N_{R}, 1} & H_{N_{R}, 2} & \cdots & H_{N_{R}, N_{T}}
\end{array}\right]\left[\begin{array}{c}
X_{1} \\
X_{2} \\
\vdots \\
X_{N_{T}}
\end{array}\right]+\left[\begin{array}{c}
W_{1} \\
W 2 \\
\vdots \\
W_{N_{T}}
\end{array}\right]
$$

in the above equation $\left[W_{1}, W_{2} \ldots \ldots W_{\mathrm{NT}}\right]$ denotes AWGN and $H_{\mathrm{m}, \mathrm{n}}$ is the (single-input single-output) channel gain between the $\mathrm{m}^{\text {th }}$ receive and $n^{\text {th }}$ transmit antenna pair. The $n^{\text {th }}$ column of $\mathbf{H}$ is often referred to as the spatial signature of the $\mathrm{n}^{\text {th }}$ transmit antenna across the receive antenna array.

Knowing the channel information at the receiver, Maximum Likelihood (ML) detection can be used for decoding of received signals for two antenna transmission system, which can be written as

$$
\begin{aligned}
& \tilde{S}[2 k]=\sum_{i=1}^{N_{R}} H_{i, 1}^{*}[2 k] Y_{i}[2 k]+H_{i, 2}[2 k] Y_{i}^{*}[2 k+1] \\
& \tilde{s}[2 k+1]=\sum_{i=1}^{N_{R}} H_{i, 2}^{*}[2 k+1] Y_{i}[2 k]-H_{i, 1}[2 k+1] Y_{i}^{*}[2 k+1]
\end{aligned}
$$

where $k=0,1,2 \ldots \ldots(N / 2)-1$

Assuming that the channel gains between two adjacent subchannels are approximately equal. i.e.

$$
H_{i, 1}[2 k]=H_{i, 1}[2 k+1]
$$

and

$$
H_{i, 2}[2 k]=H_{i, 2}[2 k+1]
$$

At the end, the elements of block $\tilde{s}[k]$ are demodulated to extract the information data.

\section{CHANNEL ESTIMATION}

Based on those assumptions such as perfect synchronization and block fading, we end up with a compact and simple signal model for both the single antenna OFDM and MIMO-OFDM systems. In training based channel estimation algorithms, training symbols or pilot tones that are known to the receiver, are multiplexed along with the data stream for channel estimation. The idea behind these methods is to exploit knowledge of transmitted pilot symbols at the receiver to estimate the channel. For a block fading channel, where the channel is constant over a few OFDM symbols, the pilots are transmitted on all subcarriers in periodic intervals of OFDM blocks. This type of pilot arrangement, depicted in Fig. 3(a), is called the block type arrangement. For a fast fading channel, where the channel changes between adjacent OFDM symbols, the pilots are transmitted at all times but with an even spacing on the subcarriers, representing a comb type pilot placement, Fig. 3(b) The channel estimates from the pilot subcarriers are interpolated to estimate the channel at the data subcarriers.

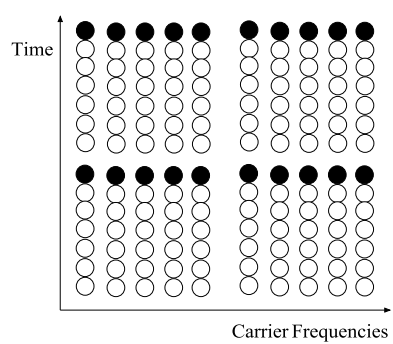

Figure 3.1. Block Pilot

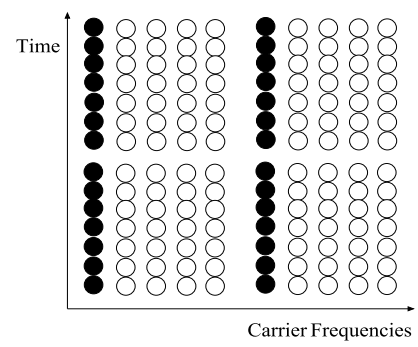

3.2. Comb Pilot

\subsection{SISO OFDM Channel Estimation}

In block-type pilot based channel estimation, OFDM channel estimation symbols are transmitted periodically, in which all subcarriers are used as pilots. If the channel is constant during the block, there will be no channel estimation error since the pilots are sent at all carriers. The estimation can be performed by using either LS or MMSE [9], [10].

In comb-type pilot based channel estimation, the $N_{p}$ pilot signals are uniformly inserted into $X(k)$ according to the following equation:

$$
X(k)=X(m L+l)
$$$$
l=1,2 \ldots . L-1
$$

Where $L=$ No. of subcarriers $/ N_{p}$ and $m$ is pilot carrier index. If inter symbol interference is eliminated by the guard interval, we write (6) in matrix notation

$$
Y=X F h+W
$$

Where 


$$
\begin{aligned}
X & =\operatorname{diag}\{X(0), X(1) \ldots \ldots . X(N-1)\} \\
Y & =[Y(0), Y(1) \ldots . . Y(N-1)]^{T} \\
W & =[W(0), W(1) \ldots \ldots . W(N-1)]^{T} \\
H & =[H(0), H(1) \ldots . . H(N-1)]^{T}=D F T\{h\} \\
F & =\left[\begin{array}{ccc}
W_{N}^{00} & \ldots & W_{N}^{0(N-1)} \\
\vdots & \ddots & \vdots \\
W_{N}^{(N-1) 0} & \ldots & W_{N}^{(N-1)(N-1)}
\end{array}\right]
\end{aligned}
$$

is the DFT matrix with

$$
W_{N}^{n k}=\frac{1}{\sqrt{N}} e^{-j 2 \pi(n / N) k}
$$

If the time domain channel vector $h$ is Gaussian and uncorrelated with the channel noise $W$, the frequency domain MMSE estimate of is given by [3]:

$$
\hat{H}_{M M S E}=F R_{h Y} R_{Y Y}^{-1} Y
$$

Where

$$
\begin{aligned}
& R_{h Y}=E\{h Y\}=R_{h h} F^{H} X^{H} \\
& R_{Y Y}=E\{Y Y\}=X F R_{h h} F^{H} X^{H}+\sigma^{2} I_{N}
\end{aligned}
$$

are the cross covariance matrix between $h$ and $Y$ and auto covariance matrix of $Y$ respectively. $R_{\mathrm{hh}}$ is the autocovariance matrix of $h, \sigma^{2}$ represents the noise variance $E\left\{|W(k)|^{2}\right\}$ and $I_{N}$ is the $N \mathrm{X} N$ Identity matrix. To use LS (least square) method for channel estimation, we usually put those obser- vation equations into a matrix form. LS is a well-known method and widely used for estimation. We choose LS rather than other methods like MMSE channel estimation for the simplicity of implementation. The LS channel estimate is represented by:

$$
\hat{H}_{L S}=X^{-1} Y
$$

the LS estimator is equivalent to what is also referred to as the zero-forcing estimator. In comb-type pilot based channel estimation, an efficient interpolation technique is necessary in order to estimate channel at data sub-carriers by using the channel information at pilot sub-carriers.

In the linear interpolation method the channel estimation at the data-carrier $k, m L<k<(m+1) L$, using linear interpolation is given by:

$$
\begin{aligned}
H_{e}(k) & =H_{e}(m L+l) \quad 0 \leq l<L \\
& =\left(H_{p}(m+1)-H_{p}(m)\right) \frac{l}{L}+H_{p}(m)
\end{aligned}
$$

Where $H_{p}$ is channel estimation value at pilot frequency. The low-pass interpolation is performed by inserting zeros into the original sequence and then applying a lowpass FIR filter that allows the original data to pass through unchanged and interpolates between such that the mean-square error between the interpolated points and their ideal values is minimized. The spline cubic interpolation produces a smooth and continuous polynomial fitted to given data points.

\subsection{MIMO OFDM Channel Estimation}

Similar to the SISO scenario, the LS channel estimation for MIMO-OFDM System between $n^{\text {th }}$ transmitter and $\mathrm{m}^{\text {th }}$ receiver antenna is

$$
\hat{H}_{L S}^{(n, m)}=\left(X^{(n)}\right)^{-1} Y^{(m)}
$$

and MMSE channel estimation for MIMO-OFDM System between $\mathrm{n}^{\text {th }}$ transmitter and $\mathrm{m}^{\text {th }}$ receiver antenna is

$$
\hat{H}_{M M S E}^{(n, m)}=F R_{h Y} R_{Y Y}^{-1} Y^{(m)}
$$

where

$$
\begin{aligned}
& R_{h Y}=R_{h h}^{(m, n)} F^{H}\left(X^{(n)}\right)^{H} \\
& R_{Y Y}=X^{(n)} F R_{h h}^{(n, m)} F^{H}\left(X^{(n)}\right)^{H}+\sigma^{2} I_{N}
\end{aligned}
$$

Where $\mathrm{n}=1,2 \ldots N_{T}, \mathrm{~m}=1,2 \ldots N_{R}$ and $N_{T}, N_{R}$ are the numbers of transmit and receive antennas, respectively, $X^{(\mathrm{n})}$ is an $N X N$ diagonal matrix whose diagonal elements correspond to the pilots of the $n^{\text {th }}$ transmit antenna and $Y^{(\mathrm{m})}$ is $\mathrm{N}$ length received vector at receiver antenna $m$.

\section{PERFORMANCE EVALUATION}

\subsection{System Parameters}

OFDM system parameters used in the simulation are indicated in Table I. We assume to have perfect synchronization since the aim is to observe channel estimation performance. Moreover, we have chosen the guard interval to be greater than the maximum delay spread in order to avoid inter-symbol interference. Simulations are carried out for different signal-tonoise (SNR) ratios. The simulation parameters to achieve those results are shown in the table. 1 .

Table 1. Simulation Parameters

\begin{tabular}{|c|c|}
\hline Parameters & Specification \\
\hline FFT Size & 128 \\
\hline No. of active Carriers & 128 \\
\hline Guard Interval & 32 \\
\hline Signal Constellation & QPSK \\
\hline Channel Model & Rayleigh Fading \\
\hline No. of Frames & 1000 \\
\hline Pilot Type & Block, comb \\
\hline
\end{tabular}




\subsection{Channel Model}

One multi-path fading channel model for SISO and 4 multipath fading channel models for 2 X 2 MIMO are used in the simulations. The channel model for SISO OFDM with sampling interval $N_{s}$ is given by:

$h(n)=\delta(n)+\delta\left(n-0.5 N_{s}\right)+\delta\left(n-3.5 N_{s}\right)$

The channel models for MIMO OFDM are given by:

$h_{11}(n)=\delta(n)+\delta\left(n-0.5 N_{s}\right)+\delta\left(n-3.5 N_{s}\right)$

$h_{12}(n)=\delta(n)+\delta\left(n-0.4 N_{s}\right)+\delta\left(n-1.1 N_{s}\right)$

$h_{21}(n)=\delta(n)+\delta\left(n-0.4 N_{s}\right)+\delta\left(n-0.9 N_{s}\right)$

$h_{22}(n)=\delta(n)+\delta\left(n-0.6 N_{s}\right)+\delta\left(n-2.2 N_{s}\right)$

We can observe performance increase in multiplexing gain of MIMO system by examining the capacity vs SNR and the increased diversity gain by means of the plot of MSE and BER. The capacity plot which deriving multiplexing gain of SISO and MIMO systems is shown in Fig. 4. And we can examine increased diversity gain in MIMO System by means of Mean Square Error (MSE) as shown in Fig. 5 and 6 and also by means of Bit Error Rate (BER) as shown in Fig. 7,8 9,10. Fig. 4 shows capacity plot of both SISO and 2 X 2 MIMO systems. In that figure we can observe that, we should have $3 \mathrm{~dB}$ more Signal power in SISO system to achieve the same data rate of MIMO system.

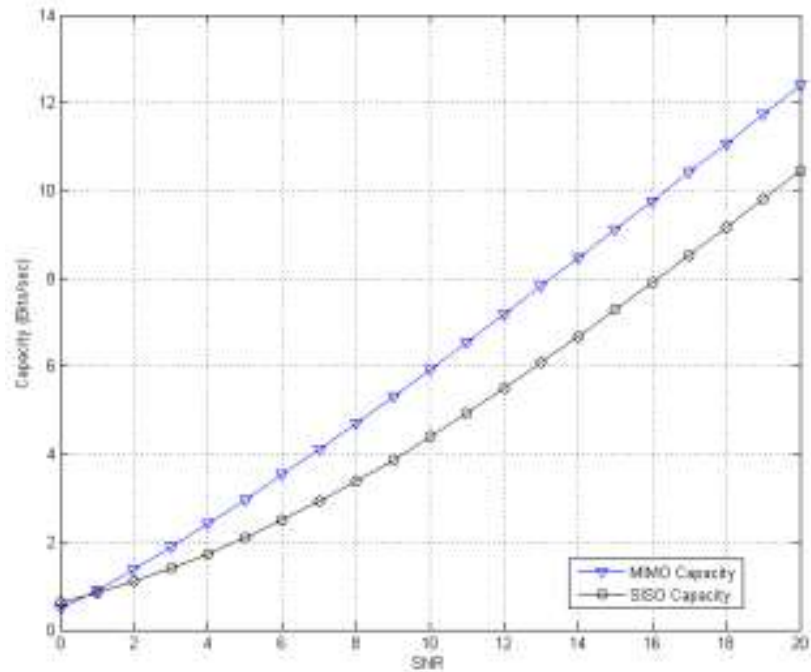

Figure 4. Capacity vs. SNR for SISO/MIMO OFDM Systems
We have modeled block-type and comb type pilot based channel estimations. Each block consists of a fixed number of symbols, which are 100 in the simulation. Pilots are sent in all the sub-carriers of the first symbol of each block in block-type model and pilots are sent in each symbol with some period in comb type and finally channel estimation is performed by using LS and MMSE estimation.

\subsection{Simulation Results and Discussions}

The instantaneous MSE is defined as the average error within an OFDM block and that can be expressed as (19), wheree $k$ is the index of the sub carries and $H_{\mathrm{e}}(k)$ is estimated value of channel attenuation.

$$
M S E=\frac{1}{N} \sum_{k=1}^{N}\left|H(k)-H_{e}(k)\right|^{2}
$$

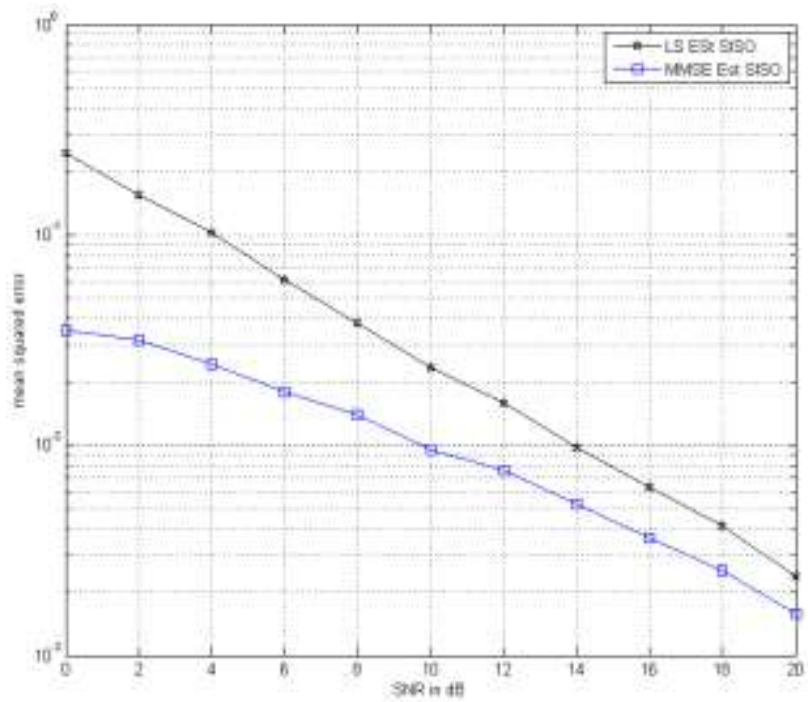

Figure 5. MSE for LS/MMSE Estimators to SISO OFDM Systems 


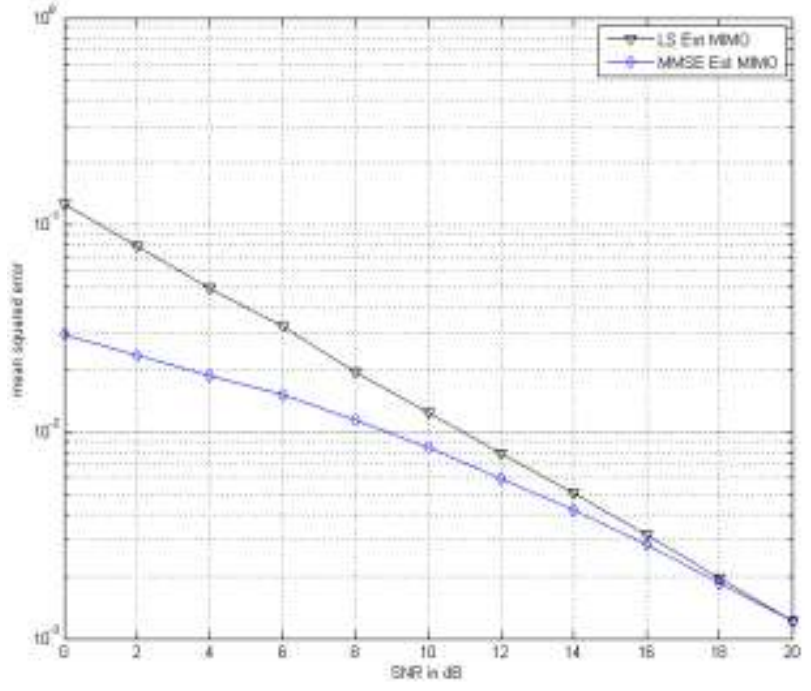

Figure 6. MSE for LS/MMSE Estimators to MIMO OFDM Systems

The mean square error of channel is defined as the mean square error of frequency demine channel response. Fig. 5 and Fig. 6 shows the MSEs of the LS and MMSE channel estimation algorithms of SISO-OFDM and MIMO OFDM systems respectively.

Fig. 5 and Fig. 6 shows the mean MSE versus the SNR, which is averaged over 1000 OFDM blocks at every SNR value. It can be observed that the performance improvement is there to system by system and also estimation algorithm wise. Out of all the above plots MIMO OFDM MMSE estimation constitutes low MSE.

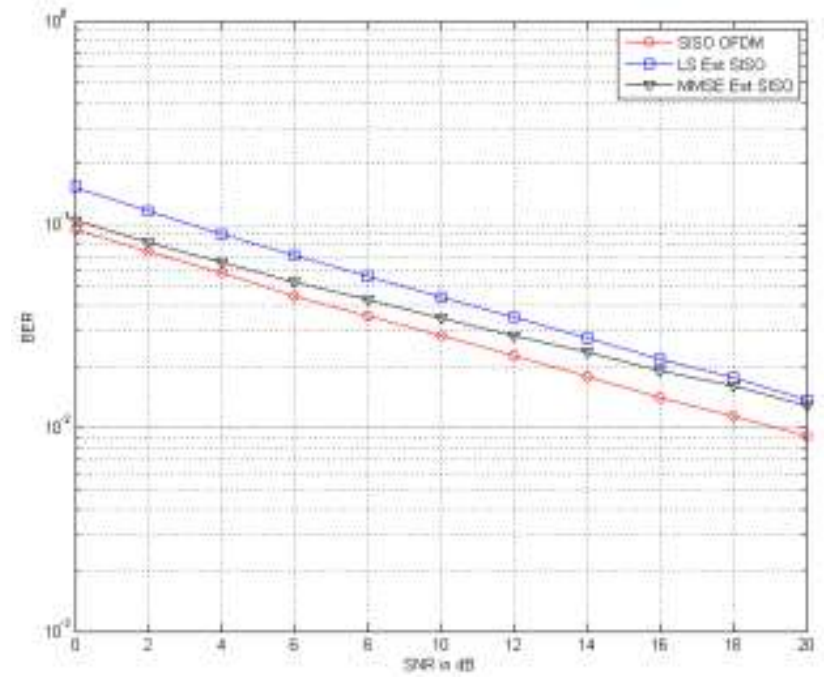

Figure 7. BER plot for Block type Pilot based SISO OFDM with LS/MMSE Estimators

From Fig. 7 denotes BER plots of the SISO system when channel is known to receiver and with LS and MMSE estimated channels. The BER performance when channel is perfectly known at the receiver is taken as the reference. Our aim is to get a result which is as close as possible to that known channel curve. Out of remaining two plots MMSE plot have fewer error than LS plot. As seen in Fig. 7, a gain in SNR up to $3 \mathrm{~dB}$ can be obtained for certain SNRs when using a MMSE estimator instead of the LS estimator.

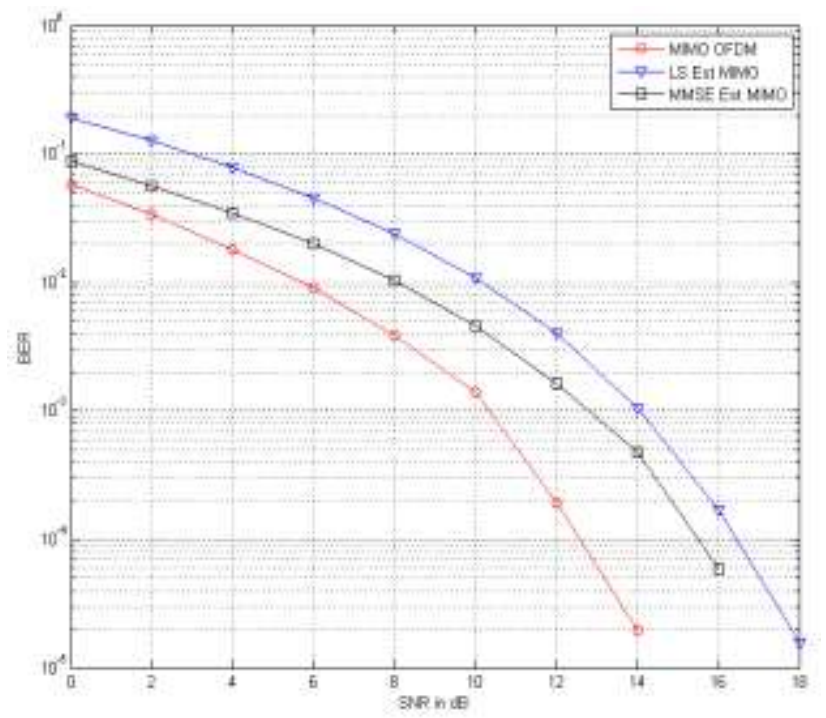

Figure 8. BER plot for Block type pilot based MIMO OFDM with LS/MMSE Estimators

From Fig. 8 denotes BER plots of the MIMO system when channel is known to receiver and with LS and MMSE estimated channels. As SISO system in Fig. 8 the BER performance of known channel is compared to the channel estimator with LS based algorithm and that of with MMSE method. From Fig.7 we can observe that there is a $\mathbf{5} \mathrm{dB}$ loss with LS estimator and there is a $\mathbf{2} \mathrm{dB}$ loss with MMSE estimator when it compares with ideal case. From Fig. 7 and Fig. 8 we can examine the discrepancy between SISO and MIMO systems with respect to BER performances. In SISO OFDM we can achieve 0.035 BER at 8 dB SNR but in MIMO OFDM system we can achieve 0.035 BER at $2 \mathrm{~dB}$ SNR itself. So there is significant SNR gain of $6 \mathrm{~dB}$.

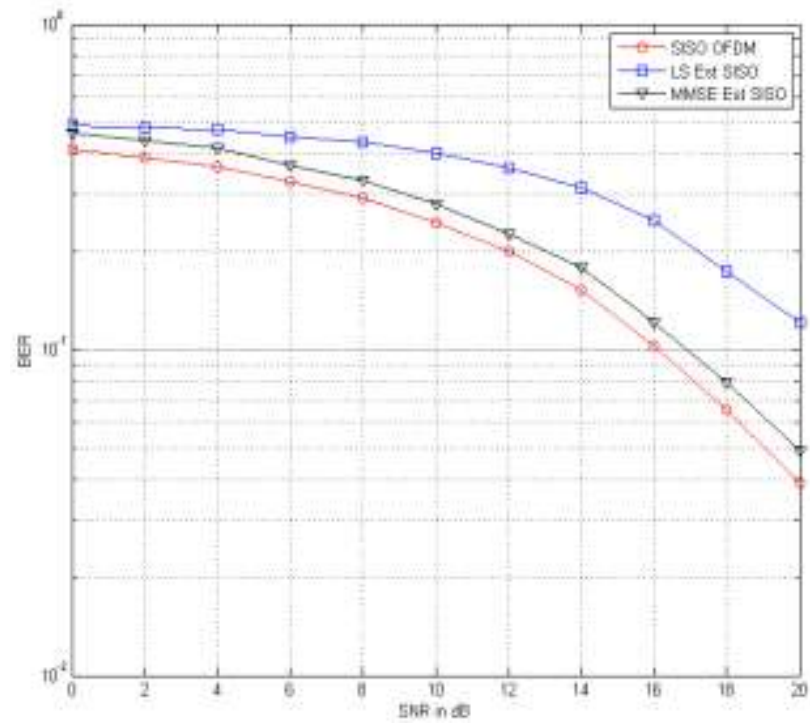

Figure 9. BER plot for comb type SISO OFDM with LS/MMSE Estimators 


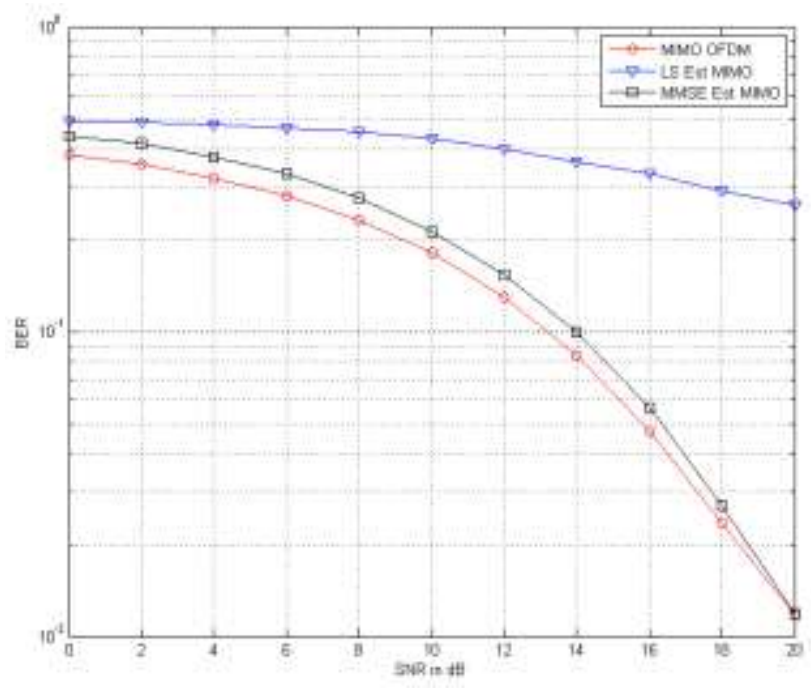

Figure 10. BER plot for comb type MIMO OFDM with LS/MMSE Estimators

However the comb-type channel estimation with low pass interpolation which achieves the best performance among all the interpolation techniques is shown in fig. 9 and Fig. 10. From Fig. 7 to Fig. 9 the BER performance of comb type pilot and block type pilot methods are compared and comb type methods are results poor performance than block type pilot based methods for the reason that of fast fading channels.

\section{CONCLUSION}

In this paper channel estimation based on both block-type pilot and comb-type arrangements in both SISO and MIMO OFDM based systems are compared. Channel estimation based on comb-type pilot arrangement is achieved by giving the channel estimation methods at the pilot frequencies and the interpolation of the channel at data frequencies. The estimators in this study can be used to efficiently estimate the channel in both OFDM systems given certain knowledge about the channel statistics. The MMSE estimator assumes a priori knowledge of noise variance and channel covariance. We then discussed Space Time Block coding and maximum likelihood decoding in the
MIMO OFDM system to enhance its performance further. We can also observe the advantage of diversity in MIMO system results less BER than SISO system. And simulation results show that MMSE estimation for MIMO OFDM provides less MSE than other systems. Finally, by comparing the performance of MMSE with LS, it is observed that the former is more resistant to the noise in terms of the channel estimation

\section{REFERENCES}

[1] Ramjee Prasad, "OFDM for Wireless Communications Systems", Artech House, Inc. Publications.

[2] Ezio Biglieri, Robert Calderbank, Robert Calderbank, Anthony Constantinides, Andrea Goldsmith, Arogyaswami Paulraj, H. Vincent Poor, "MIMO Wireless Communications", Cambridge Press.

[3] A. Petropulu, R. Zhang, and R. Lin, "Blind OFDM channel estimation through simple linear pre-coding", IEEE Transactions on Wireless Communications, vol. 3, no.2, March 2004, pp. 647-655.

[4] Osvaldo Simeone, Yeheskel Bar-Ness, Umberto Spagnolini, "Pilot-Based Channel Estimation for OFDM Systems by Tracking the Delay-Subspace", IEEE Transactions on Wireless Communications, Vol. 3, No. 1, January 2004.

[5] D. Mavares Terán, Rafael P. Torres, " Space-time code selection for OFDM-MISO system", ELSEVIER journal on Computer Communications, Vol. 32, Issue 3, 25 February 2009, Pages 477-481.

[6] Siavash M. Alamouti, "A Simple Transmit diversity Technique for Wireless Communi- cations", IEEE Journal on Select Areas in Communications, Vol. 16, No. 8, October 1998.

[7] PAN Pei-sheng, ZHENG Bao-yu, "Channel estimation in space and frequency domain for MIMO-OFDM systems" ELSEVIER journal of China Universities of Posts and Telecommunications, Vol. 16, No. 3, June 2009, Pages 4044.

[8] Mohammad Torabi, "Antenna selection for MIMO-OFDM Systems" ELSEVIER journal on Signal Processing, Vol. 88, 2008, Pages 2431-2441.

[9] J.-J. van de Beek, O. Edfors, M. Sandell, S. K. Wilson, and P. O. Borjesson, "On channel stimation in OFDM systems," in Proc. IEEE $45^{\text {th }}$ Vehicular Technology Conf., Chicago, IL, Jul. 1995, pp. 815-819. 